\title{
PREFERENCIA ALIMENTAR (ENTRE SANGUE HUMANO E AVE) DOS TRIATOMA SORDIDA ENCONTRADOS EM CASAS HABITADAS DA REGIÃO NORTE DO ESTADO DE SÃO PAULO - BRASIL
}

Eduardo Olavo da Rocha e Silva* José Maria Pacheco de Souza** José Carlos Rehder de Andrade *** Cassio da Silva Mello*** Octávio Alves Ferreira***

Rocha E Silva, E. O. et al. Preferência alimentar (entre sangue humano $e$ ave) dos Triatoma sordida encontrados em casas habitadas da região norte do Estado de São Pallo - Brasil. Rev. Saúde públ., S. Paulo, 11 :258-69, 1977.

RESUMO: São apresentados os resultados dos testes de precipitina contra soro anti-sangue humano e anti-sangue ave, realizados com 550 exemplares do T. sordida, capturados nas casas habitadas das Regionais: Ribeirão Preto e São José do Rio Preto. A partir dos percentuais de positividade para sangue humano $e$ de ave e da freqüencia relativa dos vertebrados nas casas, levantaram-se os indices de seletividade hematofilica. Como observação complementar, montou-se, no laboratório, um sistema de oferta simultânea de sangue humano e ave à ninfas do 10 estádio, não alimentadas, testando-se 185 exemplares. Concluitu-se que o T. sordida, nas áreas consideradas, suga mais ave do que homem, mesmo quando encontrado em casas habitadas. Em conseqüencia, comentam-se as possiveis implicaçōes do fato com a transmissão da endemia chagásica ao homem e com as medidas de controle.

UNITERMos: Tripanossomiase americana, profilaxia. Triatoma sordida. Triatomineos, preferência alimentar.

\section{INTRODU $\mathrm{T} O$}

Forattini e col. 6 (1974) observaram que, no Estado de São Paulo, o $T$. sordida se distribui por amplas áreas, destinadas, predominantemente, às atividades agrícolas $\mathrm{e}$ de pecuária, tendo menor significado seu encontro em áreas de maior desenvolvimento industrial.

Esses autores, levando em conta a atual divisão administrativa do Estado e os dados obtidos pela procura de triatomíneos nas

* Da Superintendência de Controle de Endemias (SUCEN) da Secretaria da Saúde do Estado de São Paulo - Rua Tamandaré, 649 - São Paulo, SP - Brasil e do Departamento de Epidemiologia da Faculdade de Saúde Pública da USP - Av. Dr. Arnaldo, 715 - São Paulo, SP - Brasil.

* Do Departamento de Epidemiologia da Faculdade de Saúde Pública da USP.

*** Da Superintendência de Controle de Endemias (SUCEN) 
ROCHA E SILVA, E. O. et al. - Preferencia alimentar (entre sangue humano e ave) dos Tratoma sordida encontrados em casas habitadas da região norte do Estado de São Paulo Brasil. Rev. Saúde públ., S. Paulo, 11:258-69, 1977.

casas, realizada anualmente pela Superintendência de Controle de Endemias (SUCEN), consideraram que são as regiões do planalto paulista, situadas na faixa de clima favorável e com alteraçōes notáveis na cobertura vegetal, processadas pela atividade humana que propiciam condiçōes para maior aproximação do $T$. sordida dos ecótopos artificiais. Vale ressaltar que a colonização de uma espécie em diferentes ecótopos expõe os exemplares a novas fontes alimentares, sendo, pois, função da amplitude da valência ecológica, sua maior ou menor capacidade de sobrevivência no novo ambiente (Forattini e col. $3,4,5,6,7$ ).

Tendo em vista o exposto, bem como " problema criado pela colonização do $T$. sordida nas casas e suas implicaçōes como possivel transmissor do Trypanosoma cruzi aos moradores, parece oportuna a realização de estudos referentes ao comportamento alimentar dos exemplares, quando presentes nas casas habitadas das regiões favoráveis, no caso, Regional 6, Ribeirão Preto e Regional 8, São José do Rio Preto (Fig. 1).

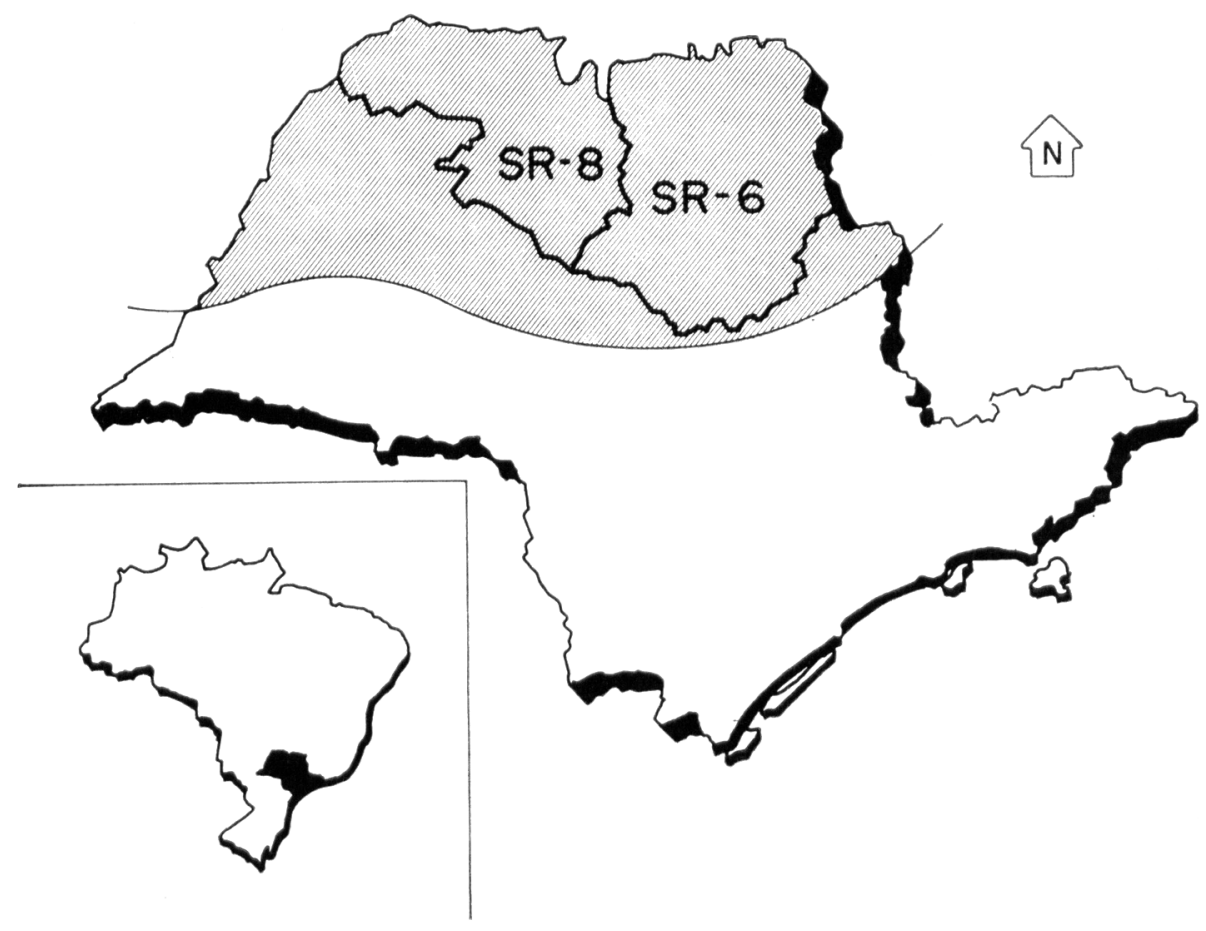

Área de clima favorāvel (segundo Forattini etal. 1974)

SR-6 - Serviço Regional 6 - RIBEIRAO PRETO

SR-8 - Serviço Regional 8 - SAOO JOSE DO RIO PRETO

Fig. 1 - Área de clima favorável à distribuição do T. sordida, no estado de São Paulo 
ROCHA E SILVA, E. O. et al. - Preferencia alimentar (entre sangue humano e ave) dos Triatoma sordida encontrados em casas habitadas da região norte do Estado de Sảo Paulo Brasil. Rev. Saúde públ., S. Paulo, 11:258-69, 1977.

Os primeiros estudos a respeito, levados a efeito em nosso meio, foram os de Corrêa e Aguiar ${ }^{2}$ (1952) e Freitas e col.* (1960). O primeiro estudando o T. infestans e o segundo realizando investigações sobre o $T$. sordida, encontrado nas dependências da Faculdade de Medicina de Ribeirão Preto. Os resultados desses últimos autores, quanto às ninfas e alados capturados nas casas e laboratórios, mostraram positividade apenas para soro anti-ave, embora testados diante de uma bateria de onze anti-soros, dentre eles $o$ anti-sangue humano.

Como hipótese para o presente estudo, considerou-se que as diferenças encontradas no decorrer dos últimos 8 anos, nos índices de infestação das casas pelo $T$. sordida (Fig. 2), infecção dos exemplares pelo $T$. crutzi (Fig. 3) e sua aglomeração nas casas (Fig. 4) ${ }^{10}$ - observadas nas citadas regio- nais - decorreram em parte de diferenças no andamento e na amplitude do trato da terra. Essas diferenças, permitiram supor também a ocorrência de defasagem no processo e na intensidade da invasão dos ecótopos artificiais pelo $T$. sórdida, nas duas áreas e conseqüentemente, no comportamento alimentar dos exemplares encontrados nas casas. Assim sendo, procurou-se, neste trabalho, estabelecer comparações entre os indices de preferência alimentar em relação ao sangue humano e ave, dos $T$. sordida capturados nas casas.

Os dados obtidos e as considerações resultantes valem também como contribuição à avaliação das medidas de controle, tendo em vista que o $T$. infestans, principal transmissor, já foi praticamente eliminado, sendo o $T$. sordida atualmente a espécie predominante nas casas dessas áreas.

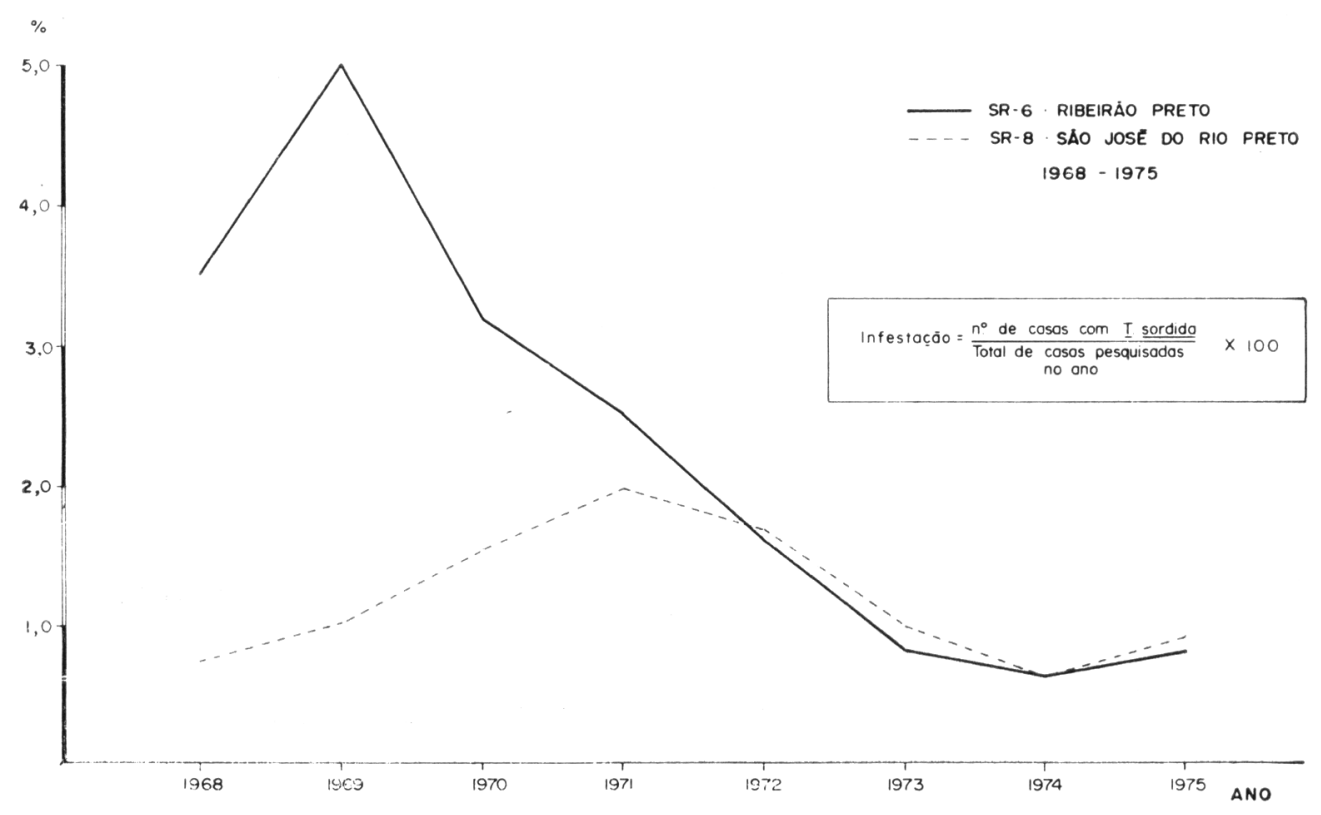

Fig. 2 - Indices de infestação das casas habitadas, pelo $T$. sordida, segundo os anos e as

Fonte: SUCEN (SR-6 e SR-8) regionais. 
ROCHA E SILVA, E. O. et al. - Preferência alimentar (entre sangue humano e ave) dos Triatoma sordida encontrados em casas habitadas da região norte do Estado de São Paulo Brasil. Rev. Saúde públ., S. Paulo, 11:258-69, 1977.

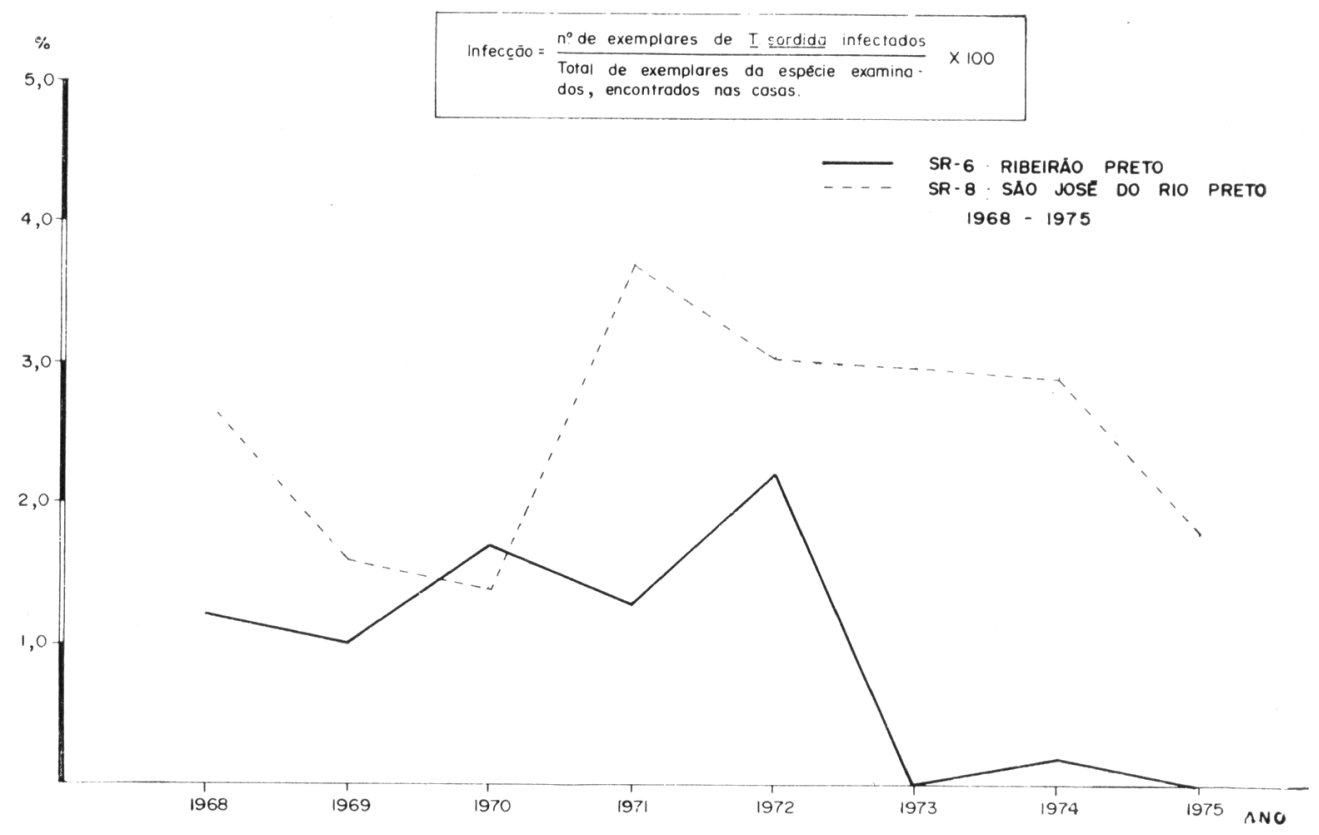

Fig. 3 - fndices de infeção pelo T. cruzi, dos $T$. sordida, segundo os anos e as regionais. Fonte: SUCEN (SR-6 e SR-8)

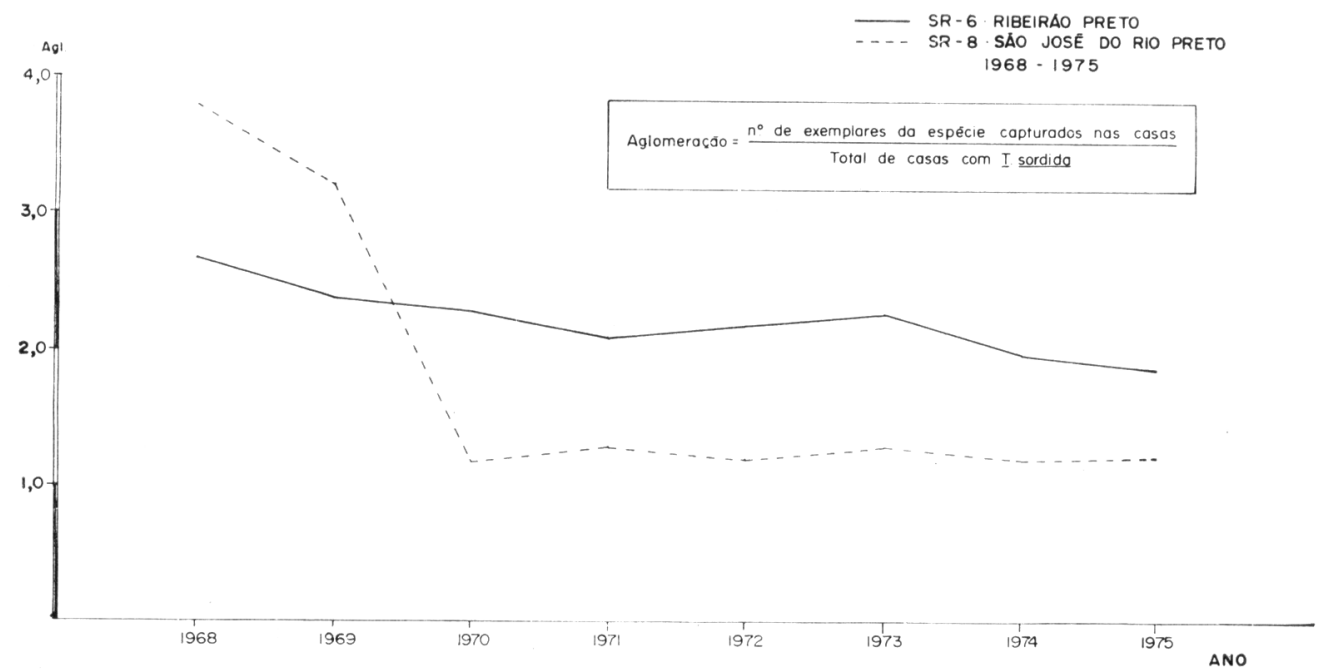

Fig. 4 - tndices de aglomeração do $T$, sordida, nas casas habitadas, segundo os anos e as Fonte: SUCEN (SR-6 e SR-8) 
ROCHA E SILVA, E. O. et al. - Preferencia alimentar (entre sangue humano e ave) dos Triatoma sordida encontrados em casas habitadas da região norte do Estado de São Paulo Brasil. Rer. Saúde públ., S. Paulo. 11:258-69, 1977.

\section{MATERIAL E MÉTODOS}

Visando detectar a presença de sangue humano e/ou de ave, no tubo intestinal dos exemplares de $T$ sordida, capturados nas casas habitadas, situadas em municipios pertencentes às regionais de Ribeirão Preto (SR-6) e de São José do Rio Preto (SR-8), foram efetuadas, no decorrer de 1975, tarefas de campo e laboratório, pela SUCEN. No caso específico do SR-6 (Ribeirão Preto), tendo en vista elevar o número das amostras, foram incluidas aincla amostras disponiveis, colhidas no primeiro trimestre de 1976.

Da Regional 6, Ribeirāo Preto, foram examinados 155 exemplares de $T$. sordida, capturados em 112 casas distribuidas por 18 dos 80 municípios que compōem o SR-6. Da Regional 8, São José do Rio Preto, foram examinados 395 exemplares provenientes de 47 municinios do total de 85 que compõem o SR-8. A suma das amostras oriundas das citadas Regionais, representaram aproximadamente $85 \%$ dos $T$. sordida capturados nas casas, no Estado, no período considerado.

As reações de precipitinas, realizadas com material intestinal colhido em papel de tiltro, foram feitas pela técnica dos tubos capilares, de Siqueira ${ }^{13}$, seguindo-se recomendação de estudo comparativo recém publicado (Andrade e col. ${ }^{1}$ ). Os anti-surus utilizados foram preparados segundo Weitz $^{1 \pm}$, com modificaçós de Siqueira ${ }^{1 .:}$

Para estabelecimento dos indices de seletividade hematofilica, segundo orientação de Hess $t$ col. $v$, estabeleceu-se a freqüência relativa dos vertebrados presentes nas casas à noite, nas duas áreas (Tabela 1).

'I' A B E L A 1

Distribuição do número médio de veltebrados e sua frequência relativa nas habitações da zona rural, das Regionais: Ribeirão Preto e São José do Rio Preto, 1975

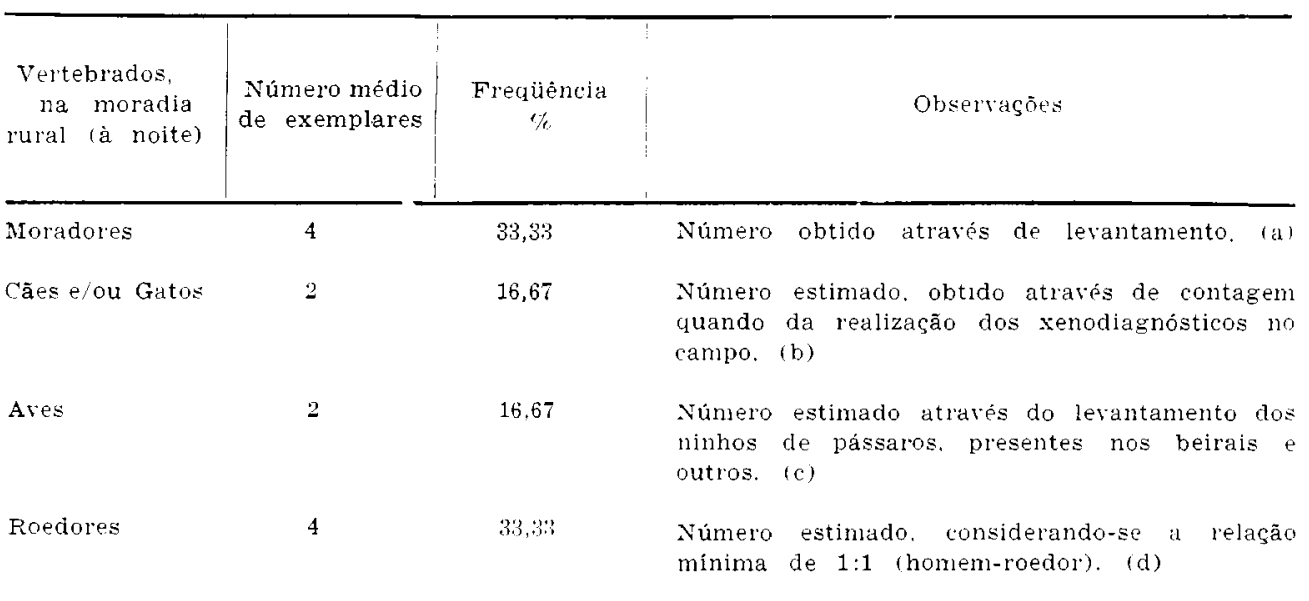

TOTAL 12

a) nas atividades de campo da SUCEN.

b) realizados pela SUCEN.

c) através dos boletins informativos dos locais de capturia dos triatomíneos (SUCEN).

d) Extraído do Seminário sobre Situação Saúde nas Áreas Metropolitanas Brasileiras,

Tema IX - Saúde ambiental. São Paulo, 1975. 
ROCHA E SILVA, E. O. et al. - Preferencia alimentar (entre sangue humano e ave) dos Triatoma sordida encontrados em casas habitadas da região norte do Estado de São Paulo Brasil. Rev. Saũde públ., S. Paulo, 11:258-69, 1977.

Como observação complementar, o laboratório passou a receber ovos do T. sordida, coletados nas duas regionais. Devidamente separados, aguardava-se a eclosão, para depois de alguns dias, serem oferecidos às ninfas do 1 " estádio, num total de 185 , como primeiro repasto alimentar, simultaneamente, a igual distância e com liberdade de escolha e deslocamento, sangue humano (antebraço) e sangue de ave (pomba). Para a efetivação deste experimento, montou-se dispositivo especial, tipo canaleta, de madeira com paredes laterais de vidro $(34 \times 5 \mathrm{~cm})$, tendo no centro um orificio de $5 \mathrm{~cm}$ de diâmetro que, no momento do teste, recebe uma caixa de papelão de igual tamanho com as ninfas. Nas extremidades livres, situadas a igual distância $(17 \mathrm{~cm})$, ajustam-se por sorteio, de um lado um antebraço humano e do outro, o peito depenado de uma pomba (Figura 5). No escuro, durante 45 min., com vela permanentemente acesa e/ou lanterna de mão intermitentemente ligada, acompanhou-se o deslocamento das ninfas até a efetivação do repasto sanguíneo. Na medida em que se engorgitavam e abandonavam a fonte alimentar, as ninfas eram retiradas e contadas em separado, considerando-se indiferentes aquelas que não abandonaram a caixa em busca do alimento ou que em se deslocando, não realizaram repasto.

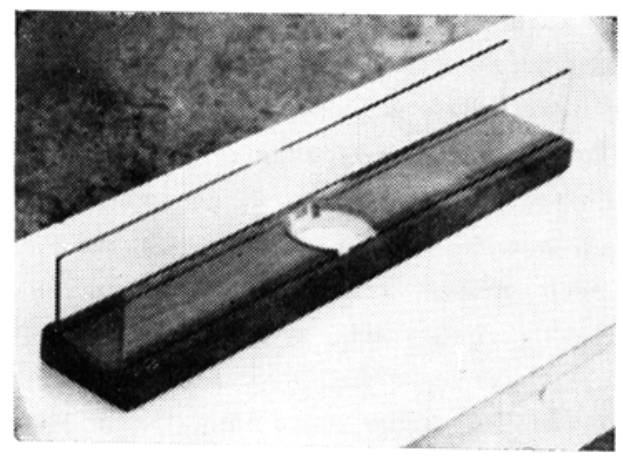

Fig. 5 -- Dispositivo de alimentação das ninfas, no laboratório.
As análises estatísticas foram feitas a partir de tabelas de associação, comparando-se proporções entre populações correlatas, através do teste do sinal, exato ou aproximado de Mc Nemar ${ }^{1:}$ (com correção) de Yates para continuidade), de acordo com o tamanho da amostra. O nivel de significância foi de $5 \%$, para teste monocaudal.

\section{RESULTADOS E DISCUSSÃO}

\subsection{Reações de Precipitina}

Os exemplares foram grupados segundo - local de captura, nos seguintes extratos que, de certa forma, medem um afastamento crescente do elemento humano como fonte de sangue para alimentação:

a) captura na cama;

b) captura no quarto, mas não na cama; c) captura fora do quarto.

É importante lembrar que todos os exemplares foram capturados em casa habitada, cada um deles podendo apresentar qualquer uma das quatro possíveis combinações quanto à presença de sangue humano e de ave (humano positivo $e$ ave positivo; humano positivo e ave negativo; humano negativo e ave positivo; humano negativo e ave negativo).

As Tabelas 2, 4, 6 e 8 apresentam os resultados dos exames de precipitina realizados nos triatomíneos capturados na regional de Ribeirão Preto e as de número 3,5 , 7 e 9 na regional de São José do Rio Preto. Os resultados das reaçóes com os exemplares capturados fora da (ama e fora di) quarto (Tabelas 4, 5, 6 e 7), mostram que há preferência por sangue de ave, tendo os testes estatísticos sido significantes para a hipótese alternativa $\left(\mathrm{H}_{0}\right.$ : preferência por ave $=$ preferência por humano; $\mathrm{H}_{\mathrm{A}}$ preferência por ave $>$ preferência por humano).

Chama atenção o resultado para exemplares capturados na cama (Tabelas 2 e 3 ) quando a preferência pela fonte alimentar foi marcadamente diferente das demais tabelas. 
ROCHA E SILVA, E. O, et al. - Preferência alimentar (entre sangue humano e ave) dos Triatoma sordida encontrados em casas habitadas da região norte do Estado de São Paulo Brasil. Rev. Saúde públ., S. Paulo, 11:258-69, 1977.

Assim, em São José do Rio Preto, há equilibrio quanto aos resultados da precipitina para sangue humano e sangue de ave (aceita-se $\mathrm{H}_{0}$ ). Em Ribeirão Preto, a diferença de comportamento é mais evidente; devido ao teste estatístico proposto ser monocaudal, não houve necessidade de ser realizado, aceitando-se também, automaticamente, a hipótese $H_{o}$, com sugestão, inclusive, de preferência por sangue humano.

Tendo em vista o exposto, reforça-se a observação de Forattini e col. " de que o $T$. sordida se aproxima das casas, não necessariamente pela presença do homem, mas sim levado pela dispersão e sobrevivência da espécie. Uma vez instalado na casa habitada, o $T$. sordida encontra no novo ecótopo, entre outros, o homem como fonte alimentar, embora continue a se alimentar em aves.

Os dados apresentados acima sugerem que a frequiência do repasto humano parece não ser igual, nas duas regionais. Entre os fatores que podem influenciar a diferença observada, supõe-se presente a defasagem no andamento das causas desencadeantes do processo de invasão das casas pelo $T$. sordida.

\subsection{Seletividade Hematofilica}

Os resultados para os indices de preferência alimentar, propostos por Hess e col. ${ }^{9}$ e mencionados em publicação da Organização Mundial de Saúde 11, encontram-se na Tabela 10 e confirmam os resultados globais obtidos através das reações de precipitina.

A preferência alimentar pelo sangue de ave revelou-se, em ambas as áreas, um pouco superior ao índice 1 , sendo maior o valor encontrado para São José do Rio Preto. Quanto aos indices de sangue humano, ambos mostram-se menores do que 1 . Recorde-se que segundo os autores, os indices abaixo de 1 , indicam preferência alimentar em favor de outros hospedeiros.

\subsection{Testes alimentares, em laboratorio}

As Tabelas 11 e 12 apresentam os resultados dos testes alimentares, realizados com ninfas de 10 estádio, não alimentadas, às quais foram oferecidas, simultaneamente, possibilidade de obter alimento a igual distância, em homem (antebraço) ou ave (peito de pomba). Foram efetivados pelo menos 4 testes com material de cada uma das Regionais, cujos resultados para facilitar a avaliação, foram somados. A hipótese do $T$. sordida apresentar preferência pelo sangue de ave em relação ao sangue humano foi aceita para ninfas procedentes de ambas as áreas. É interessante notar que a diferença menor de preferência foi das ninfas nascidas de ovos procedentes da regional de Ribeirão Preto.

\subsection{Considerą̧ōes Finais}

Os atuais baixos indices de infestação das casas pelo $T$. sordida e de infecção dos exemplares pelo $T$. cruzi (Figs. 2 e 3 ) e a aparente preferência por sangue de ave (Tabelas 4, 5, 6 e 7), sugerem que a probabilidade de ocorrência, no momento, de transmissão da doença de Chagas ao homem, nessas áreas, pode ser considerada pequena. Fato, aliás, já notado pelo esporádico conhecimento de casos humanos agudos, por transmissão natural (via triatomíneo), ocorridos nos últimos anos, nas mesmas. 


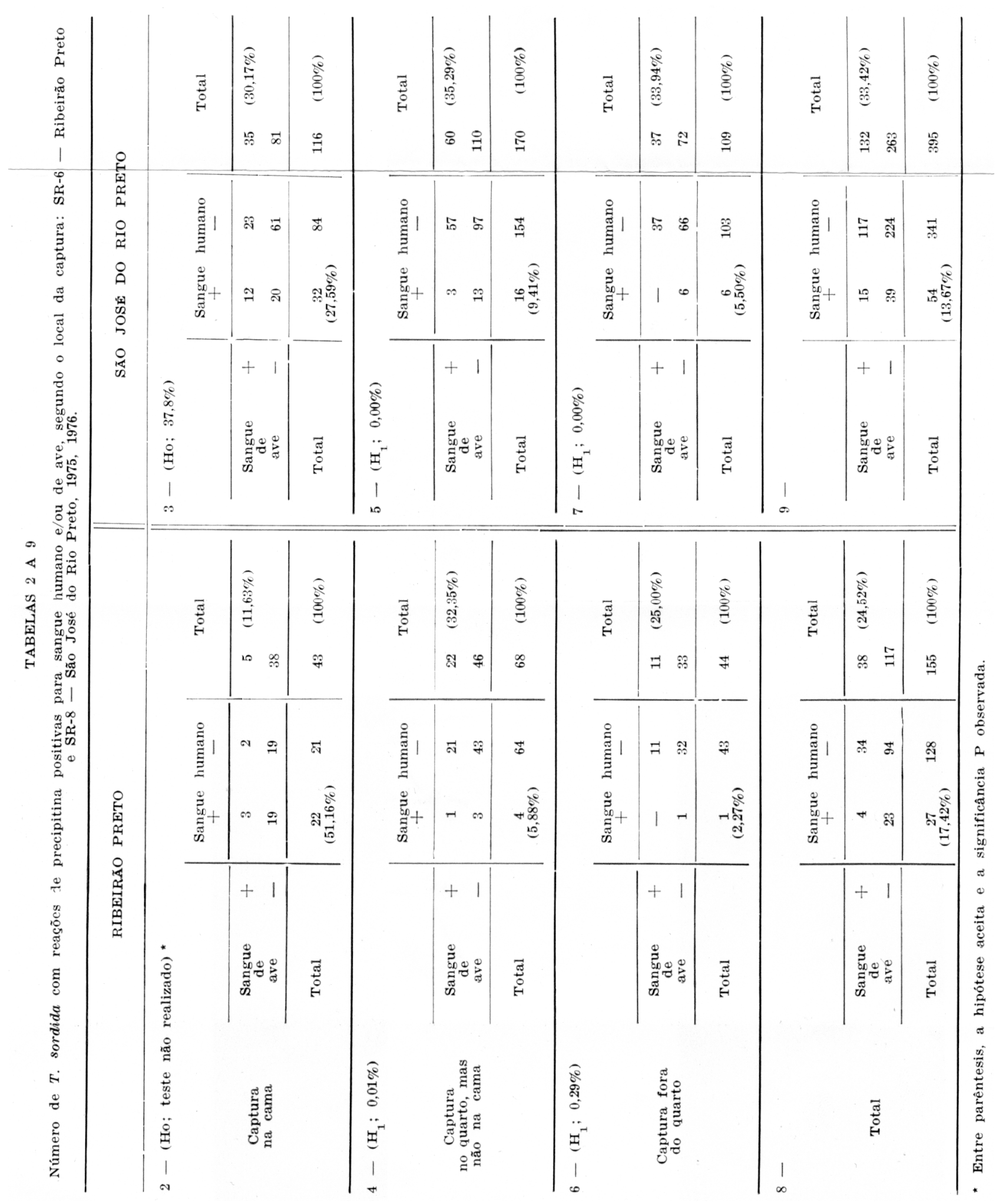


ROCHA E SILVA, E, O. et al. - Preferencia alimentar (entre sangue humano e ave) dos Triatoma sordida encontrados em casas habitadas da região norte do Estado de São Paulo Brasil. Rev. Saúde públ., S, Paulo, 11:258-69, 1977.

TABELA 10

Percentagem de $T$. sordida alimentados nas fontes humana e ave; distribuição relativa nas casas dos representantes das fontes de alimentação e indices de preferência alimentar, segundo as Regionais: Ribeirão Preto e São José do Rio Preto, 1975.

\begin{tabular}{|c|c|c|c|c|}
\hline & \multirow{2}{*}{ Regional } & \multicolumn{3}{|c|}{ Sangue humano } \\
\hline & & $\begin{array}{l}\text { Exemplares } \\
\text { encontrados } \\
\text { alimentados * }\end{array}$ & $\begin{array}{c}\text { Fonte fornece- } \\
\text { dora do all- } \\
\text { mento } * \star\end{array}$ & $\begin{array}{l}\text { fndice de } \\
\text { preferencia } \\
\text { alimentar } \star * \star\end{array}$ \\
\hline & Ribeirão Preto & $17,42 \%$ & $33,33 \%$ & 0.52 \\
\hline & \multirow[t]{3}{*}{ S. José do R. Preto } & $13,67 \%$ & $33,33 \%$ & 0,41 \\
\hline & & \multicolumn{3}{|c|}{ Sangue de ave } \\
\hline & & $\begin{array}{l}\text { Exemplares } \\
\text { encontrados } \\
\text { alimentados }\end{array}$ & $\begin{array}{l}\text { Fonte fornece- } \\
\text { dora do ali- } \\
\text { mento }\end{array}$ & $\begin{array}{l}\text { fndice de } \\
\text { preferência } \\
\text { alimentar }\end{array}$ \\
\hline & Ribeirão Preto & $24,52 \%$ & $16,67 \%$ & 1,47 \\
\hline & S. José do R. Preto & $33,42 \%$ & $16,67 \%$ & 2,00 \\
\hline$*$ & \multicolumn{4}{|l|}{ Ver Tabelas 8 e 9} \\
\hline$* *$ & \multicolumn{4}{|l|}{ Ver Tabela 1} \\
\hline$* * * ;$ & \multicolumn{4}{|c|}{ fndice de preferência alimentar da Hess e col. $9,1968}$. \\
\hline
\end{tabular}

TABELA 11

Número de ninfas segundo preferência por fonte de alimentação (braço humano ou peito de ave). Ovos coletados em Ribeirão Preto, 1976.

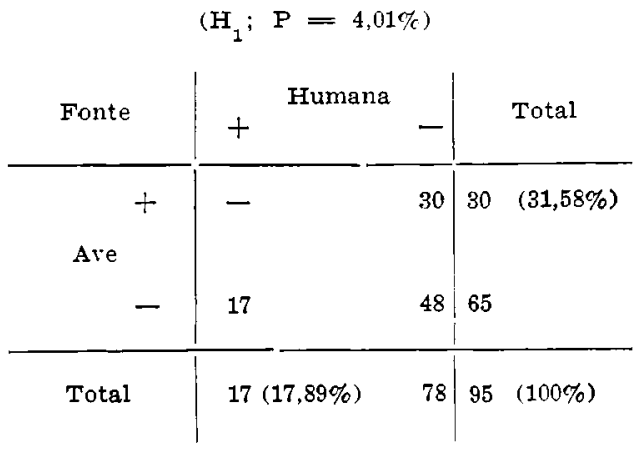

TABELA 12

Número de ninfas segundo preferência por fonte de alimentação (braço humano ou peito de ave). Ovos coletados em São José do Rio Preto, 1976.

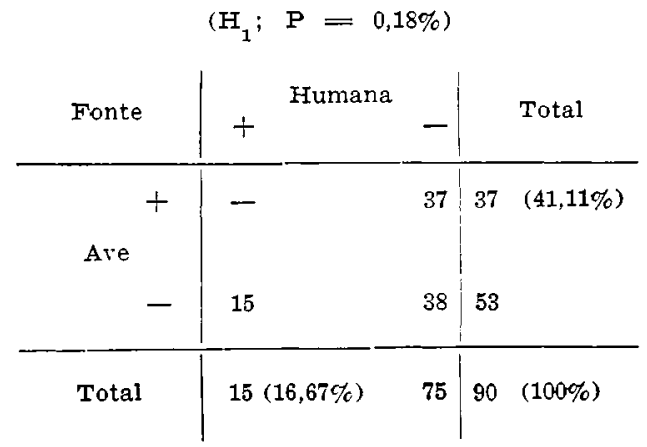


ROCHA E SILVA, E. $O$, et al. - Preferência alimentar (entre sangue humano e ave) dos Triatoma sordida encontrados em casas habitadas da região norte do Estado de São Paulo Brasil, Rev. Salide públ., S. Paulo, 11:258-69, 1977.

Os rest.ltados clas tabelas 2 e 3 , quando comparadus aus was tabelas $4,5,6,7,11 \mathrm{e}$ 12 , parecem indicar que o homem é usado como fonte de alimento, quando existem boas condições de acesso e não devido a uma real preferência alimentar. Para evitar esta aproximação, são necessárias periódicas medidas de controle, podendo ter alguma implicação a sistemática retirada dos ninhos de pássaros, especialmente no caso, pardais (Passer domesticus), dos prédios, como uma das medidas de controle adotadas. Uma hipótese a ser levantada é a de que a manutenção dos ninhos seria medida zooprofilática recomendável para áreas com $T$. sordida. O teste desta hipótese seria feito, comparando-se o comportamento dos exemplares em casas com ninhos e casas sen ninhos.

\section{CONCLUSOES}

4.1 Evidenciou-se que o $T$. sordida apresenta maior preferência pelo sangue de ave en relação ao humano.
4.2 Os resultados sugerem possiveis diferenças no comportamento alimentar do $T$. sordida, presente nas casas habitadas, comparadas às capturas realizadas nas regionais de Ribeirão Preto e São José do Rio Preto.

4.3 Levando-se em conta as informaçōes e resultados apresentados, o $T$. sordida presente nas casas habitadas, das áreas estudadas, pode ser considerado como transmissor potencial da tripanossomose americana aos moradores, porém de importância relativa.

A GRADECIMENTOS

À Biologista Sra. Vera Lucia C. Rodrigues e demais auxiliares-técnicos do laboratório da SUCEN - Moji Guaçu, bem como aos servidores das Regionais de Ribeirão Preto e São José do Rio Preto.

RSPU-B/357

Rocha E SILVA, E. O. et al. [Some considerations on the blood ingestion preference (between human or bird source) of Triatoma sordida that were found in inhabited houses, in the north regions of the State of S. Panlo - Brazil.] Rev. Saúde públ., S. Paulo, 11:258-69, 1977.

ABSTRACT: Results of preciptin tests for anti-human blood and anti-bird blood were snown for $555 \mathrm{~T}$. sordida, captured in inhabited houses in the State of S. Pallo Administrative Regions of Ribeiräo Preto and S. José do Rio Preto. Besides getting the hematophilic index of seletivity of Hess, Hayes et Tempelis ${ }^{9}$, a device built in order to offer simultaneously human blood the fore arm of the researcher) and bird blood (the breast of a pigeon) was used. The analysis of the results of preciptin tests, the index of selectivity and the results of the offering of two alternatives as blood sources, showed that the $\mathrm{T}$. sordida has a definite preference for bird blood save on two occasions, when the human source was far more available.

UnITERms: Trypanosomiasis, South American. Triatoma sordida. Triatomids, feeding preference. 
ROCHA E SILVA, E. O. et al. - Preferência alimentar (entre sangue humano e ave) dos Triatoma sordida encontrados em casas habitadas da região norte do Estado de São Paulo Brasil. Rev. Saúde pübl., S. Paulo, 11:258-69, 1977.

\section{REFERENCIAS BIBLIOGRÁFICAS}

1. ANDRADE, J. C. R. de et al. Comparação entre duas técnicas sorológicas aplicadas ao estudo do sangue ingerido por triatomíneos. Rev. Saúde puibl., S. Paulo, 9:534-45, 1975.

2. CORREA, R. R. \& AGUIAR, A. A. O teste de precipitina na identificação da fonte alimentar do Triatoma infestans (Hemiptera, Reduviidae). Arq. Hig. (S. Paulo), 17:3-7, 1952.

3. FORATTINI, O. P. et al, Aspectos ecológicos da tripanossomose americana. II - Distribuição e dispersão local de triatomíneos em ecótopos naturais e artificiais. Rev. Saúde públ, s. Paulo, 5:163-91, 1971.

4. Forattini, O. P. et al. Aspectos ecológicos da tripanossomose americana. III - Dispersão local de triatomíneos com especial referência ao Triatoma sordida. Rev. Saúde públ., S. Paulo, 5:193-205, 1971.

5. Forattini, O. $P$. et al. Aspectos ecológicos da tripanossomose americana. V - Observaçōes sobre colonização espontânea de triatomineos silrestres em ecótopos artificiais, com especial referência ao Triatoma sordida. Rev. Saúde púbi., S. Paulo, 7:219-39, 1973.

6. FORATTINI, O. P. et al. Aspectos ecológicos da tripanossomíase americana. VI - Persistência do Triatoma sordida após alteração ambiental e suas possiveis relações com a dispersão da espécie. Rev. Saúde públ., S. Paulo, 8:265-82, 1974.

7. FORATTINI, O. $P$. et al. Aspectos ecológicos da tripanossomíase americana. VII - Permanência e mobilidade do Triatoma sordida em relação a ecótopos artificiais. Rer. Saúde públ., S. Paulo, 9:467-76, 1975.

8. FREITAS, J. L. P. de et al. Investigações epidemiológicas sobre triatomineos de hábitos domésticos e silvestres com auxilio da reação de precipitina. Rev. Inst. Med. trop. S. Paulo, 2 $90-9,1960$

9. HESS, A. D. et al. The use of the forage ratio technique in mosquito host-performance studies. Mosquito News, 28:286-9, 1968.

10. JIMENEZ, J. C. Control de la enfermidad de Chagas, Caracas, Direcion de Malariologia y Saneamiento Ambiental, 1974. [Publicacion 74-03]

11. ORGANIZACION MUNDIAL DE LA SALUD. Grupo cientifico de la OMS sobre Ecologia, Ginebra, 1971. Ecologia de los vetores: informe, Ginebra 1972. [Ser. Inf. técn., 501]

12. SIEGEL, $\mathbf{S}$. Nonparametric statisc for the behavioral sciences. New York, McGraw-Hill, 1956.

13. SIQUEIRA, A. F. Estudos sobre a reação de precipitina aplicada a identificação do sangue ingerido por triatomíneos. Rev. Inst. Med. trop. $\mathbf{s}$. Paulo, 2:41-53, 1960.

14. WEITZ, B. The identification of blood meals in blood sucking arthropods by the precipitin test. Bull. Wld. Hith. Org., 15: 473-90, 1953.

Recebido para publicaşão em 22/06/1976 Aprovado para publicação em 10/09/1976 